\title{
THE DIFFERENCES IN THE DETECTABILITY OF PERFORATION SHOTS AND MICROSEISMIC EVENTS IN THE SURFACE MONITORING: THE ATTENUATION EFFECT
}

\author{
David EINŠPIGEL $^{1) *}$ and Leo EISNER ${ }^{2)}$ \\ 1) Charles University in Prague, Faculty of Mathematics and Physics, V Holešovičkách 2, 18000 Praha 8, Czech Republic \\ ${ }^{2)}$ Institute of Rock Structure and Mechanics, The Academy of Science of the Czech Republic, V Holešovičkách 41, \\ 18209 Praha 8, Czech Republic \\ *Corresponding author's e-mail: einspigel@karel.troja.mff.cuni.cz
}

\section{ARTICLE INFO}

Article history:

Received 27 February 2013

Accepted 8 November 2013

Available online 3 December 2013

Keywords:

Microseismic processing

Attenuation

Frequency domain

Detectability

\section{ABSTRACT}

Detection of the seismic signal from perforations or string shots is crucial for calibration of velocity model for surface or downhole monitoring. While in the borehole recordings perforation shots have higher signal to noise ratio of the P-waves than microseismic events, in the surface monitoring recordings it is often opposite. We investigated amplitude spectra of microseismic events and perforation shots in the downhole data and found that amplitude ratios in the band of $20-30 \mathrm{~Hz}$ relative to band $100-200 \mathrm{~Hz}$ are stronger for the microseismic events. Thus we suggest that detectability of the perforations in surface recorded data is limited by attenuation of the higher frequency signal which increases exponentially with frequency. This suggests that longer duration of calibration shots can improve detectability of string shots at the surface.

\section{INTRODUCTION}

Passive seismic monitoring relies on calibration from perforations or string shots at known positions to orient downhole $3 \mathrm{C}$ receivers (e.g., Maxwell et al., 2010) and calibrate the velocity model for surface (Duncan and Eisner, 2010) or downhole (Bulant et al., 2007 or Bardainne and Gaucher, 2010) monitoring. Hence detection of the seismic signal from the perforation or string shots is crucial to successful microseismic monitoring. Calibration shots, unlike microseismic events, are somewhat easier to detect as their position and approximate timing is known. However, there is a lack of understanding on what controls the strength of the seismic signal radiated from a perforation or string shot.

In borehole monitoring perforations or string shots usually have higher P-wave amplitudes compared to microseismic events. However, Rutledge et al. (1998) describe microseismic monitoring where a perforation shot in a dry treatment well was not detected on near downhole receivers and a subsequent shot in the same well filled with water was observed several hundred meters away. Detectability of the perforation or calibration shots seems to be even less predictable in the surface monitoring as Chambers et al. (2010) have shown in a case study that even some of the most energetic string shots are not detected while two to three times less energetic string shots are detected. In some basins microseismic events are routinely observed on surface geophones while perforations or strings shots can not be detected even after stacking. This observation poses a significant challenge as the perforation shots have higher signal- to-noise ratios (SNR) than microseismic events on downhole receivers in the very same monitoring simultaneously monitored by both downhole and surface. While detectability of perforations depends on coupling of the perforations to the formation, the challenge addressed in this study is why perforations which are observed on downhole monitoring array are not observed and even detected at the surface. Obviously in such cases coupling of perforations to formation is not an issue as seismic energy is detected on downhole recordings.

We suggest that the perforations and string shots radiate mostly high frequency energy and detectability is controlled by attenuation of the media. As attenuation increases exponentially with frequency, it reduces proportionally more high frequency signal of calibration shots. Although microseismic events may have lower SNR at high frequencies (above $100 \mathrm{~Hz}$ ) we may still detect them if they have sufficient SNR at lower frequencies. This proposed mechanism suggest that longer duration of calibration shots may have a better seismic signal at low frequencies and hence more likely to be detected by the surface monitoring.

\section{CASE STUDIES}

First we analyze downhole monitoring from Haynesville shale basin (depth exceeding $3.5 \mathrm{~km}$ ) where hydraulic fracture stimulation was monitored from both downhole vertical array as well as surface (array of shallow borehole geophones) monitoring array. As observed in the past the surface array did not detect the perforation shots even after stacking of 


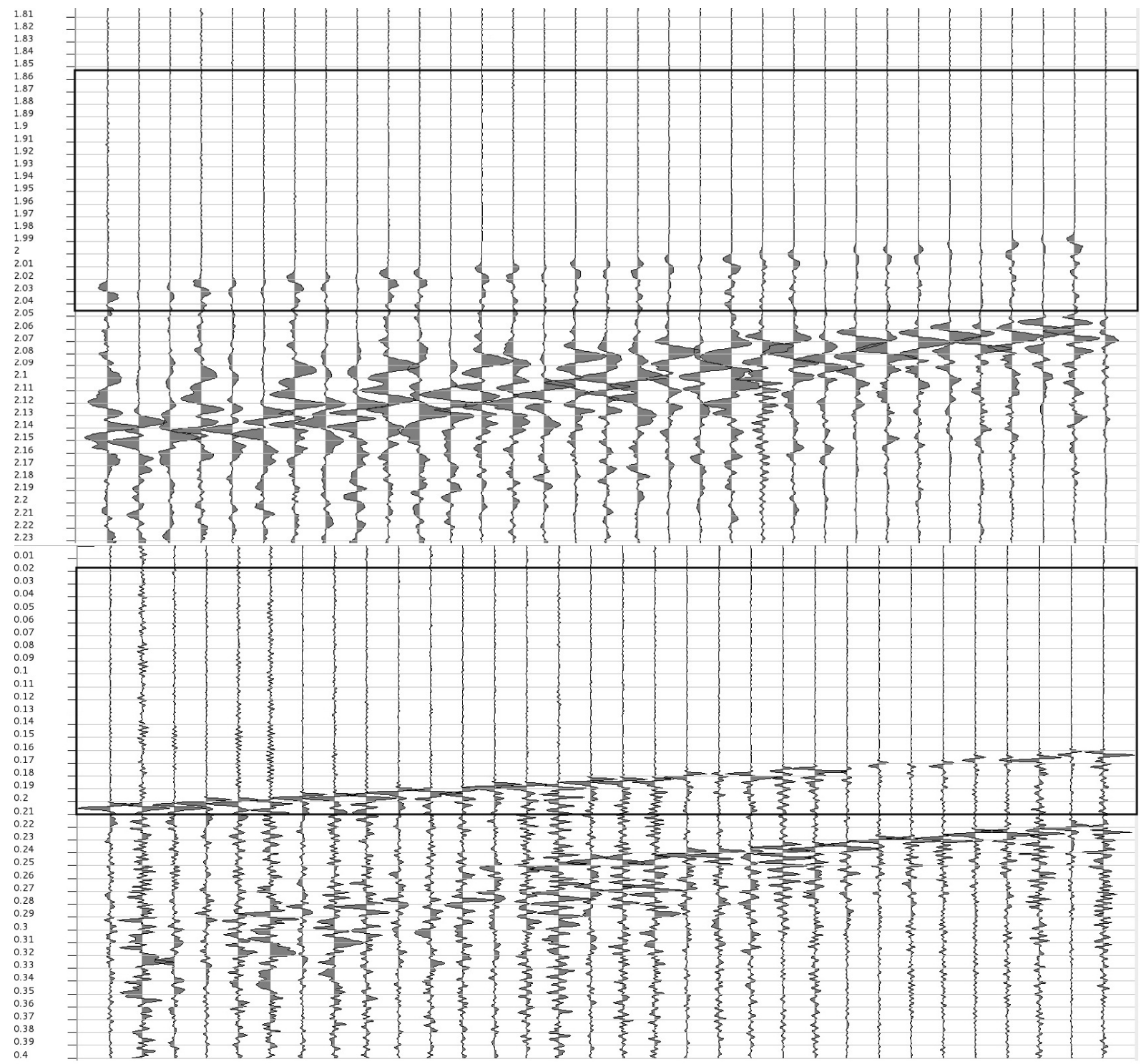

Fig. 1 Raw waveforms of particle velocity of a representative microseismic event (top panel) and perforation shot (lower panel). The black rectangle shows time interval used for Fourier Transformation around the P-wave arrival.

nearly 100 buried geophones, yet numerous microseismic events were detected including several events clearly visible on individual phones of the buried array. During stacking the attenuation was not taken into account because its value is not known. We believe it is unlikely that considering the attenuation correction before stacking would affect the detectability on the surface array, because it might significantly increase noise. Furthermore, the migration algorithm did not use true amplitudes.

We used data from the downhole monitoring array to analyze two strong microseismic events visible on individual surface geophones and two perforations to understand frequency content characteristics of the seismic signals. As mentioned above no perforation shots were detected by the surface array. The strong events were used since they have better SNR. In earthquake seismology larger magnitude $\left(M_{w}>2\right)$ events tend to give different low frequency part of the spectra because they have corner frequencies below $10 \mathrm{~Hz}$. However, in prospecting seismology we usually deal with much weaker events which have corner frequencies in the order of several hundred $\mathrm{Hz}$ or more (the weaker the event the higher the corner frequency). In our study all microseismic events have the moment magnitude less than -1 , which corresponds to the corner frequencies of $300 \mathrm{~Hz}$ and higher (Eisner et al., 2013) and below the corner frequency the amplitude spectra of particle displacement is flat down to $0 \mathrm{~Hz}$ (Aki and Richards, 2002). Based on observations, the weaker microseismic events have similar waveforms to the stronger events, i.e. similar spectra (so-called selfsimilarity, see e.g. Rutledge and Phillips, 2003). This observation result from the fact that attenuation determined the peak frequency in the observed data 


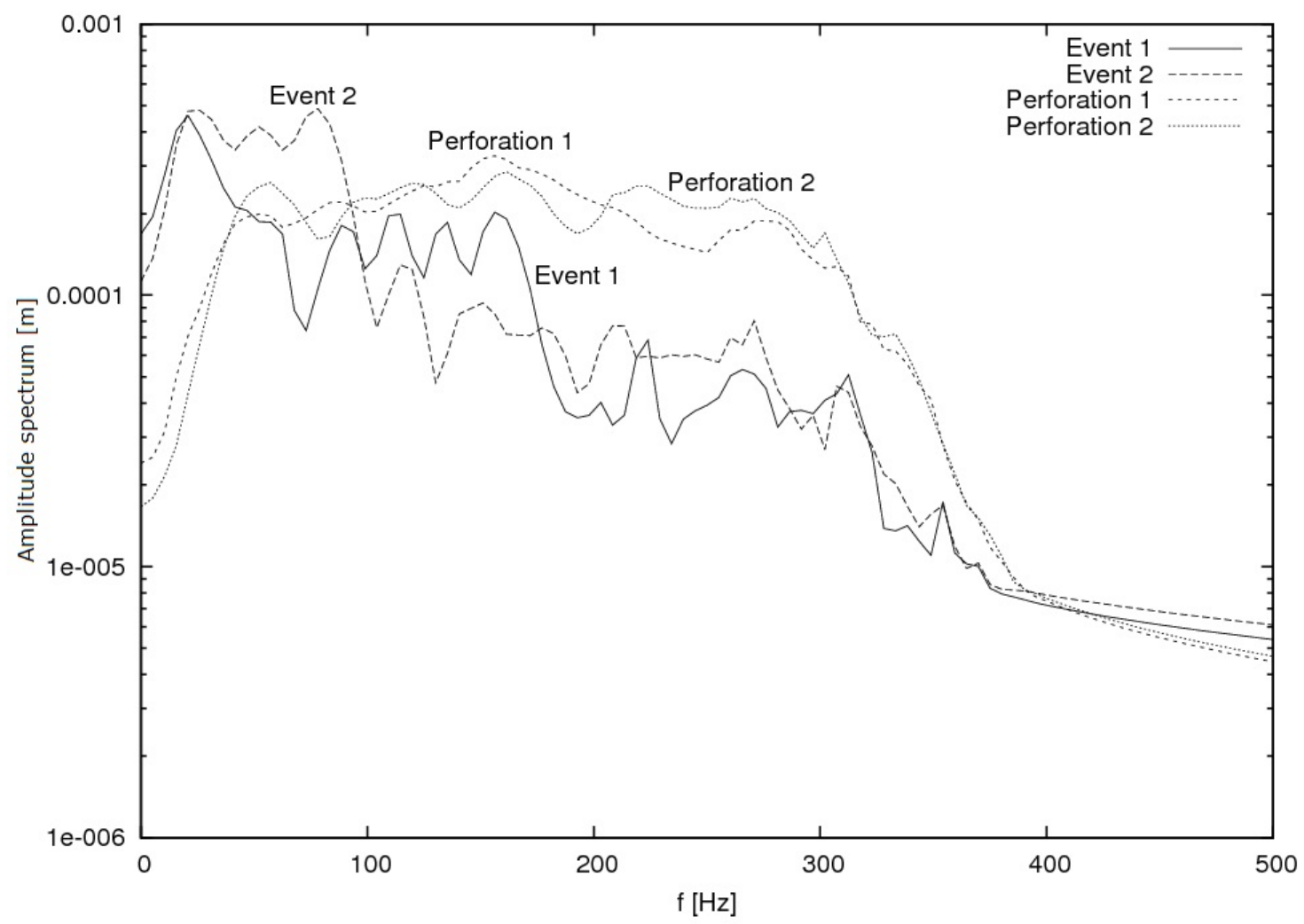

Fig. 2 Comparison of spectra of two microseismic events and two perforations from first dataset (Haynesville shale basin). Amplitude spectra are averaged over all channels of waveforms shown in Figure 1.

(Eisner et al., 2013). Hence microseismic events spectra of different magnitudes from similar locations are self-similar as observed. Hence results of our spectral analysis are not affected by the size of the studied microseismic events. We performed a fast Fourier transform of time intervals with arrivals of the $\mathrm{P}$-waves and averaged the amplitude spectra over all channels to obtain representative spectra for the event. The borehole monitoring array was approximately 300 meters above the stimulated interval hence nearly all channels sample the same part of the radiation pattern and the seismic waves travelling from the source were not significantly attenuated. Figure 1 shows representative examples of the recorded raw particle velocities of an event and a perforation shot, respectively. The sections which were used to Fourier transform are inside the black rectangle. The sections of the seismograms contain some coda after the Pwave arrival from the receivers located in higher positions, however the signals from direct P-wave arrivals are dominant in the selected window and the spectra were averaged which should eliminate any anomalies from the coda.

Figure 2 shows the averaged spectra of two events and two perforations as described above. While the absolute level of each spectral amplitude is dependent on the magnitude of the microseismic event or the size of the perforation, the relative amplitudes at different frequencies reveal important properties of the seismic signals. The spectra of two microseismic events peak below $100 \mathrm{~Hz}$ whereas the spectra of the perforations peak between 100 and $300 \mathrm{~Hz}$ (probably limited by roll-off filter) and very little energy is present below $40 \mathrm{~Hz}$. Considering that microseismic events observed at the surface geophones have usually peak amplitudes between 20 and $30 \mathrm{~Hz}$ (Duncan and Eisner, 2010) we can see that in this specific example microseismic events have 10-times larger amplitude in this frequency band. While perforations have higher signal in frequencies above $100 \mathrm{~Hz}$ such signal is more severely attenuated as attenuation increases exponentially with frequency as can be seen from amplitude dependency in attenuating medium and plane waves (Aki and Richards, 2002):

$$
A(x)=A_{o} e^{\frac{-\omega t^{* 2}}{2}}
$$

where $A(x)$ is amplitude at receiver $x, A_{0}$ is amplitude at unit distance around the source, $\omega$ is circular frequency and $t^{*}=\int_{\text {ray }} \frac{d s}{c(x) Q(x)}$, where $s$ stands for ray segment, $x$ is the position along the ray segment and $c(x)$ and $Q(x)$ are corresponding velocity and quality factor at position $x$. Note, that for 5-times 


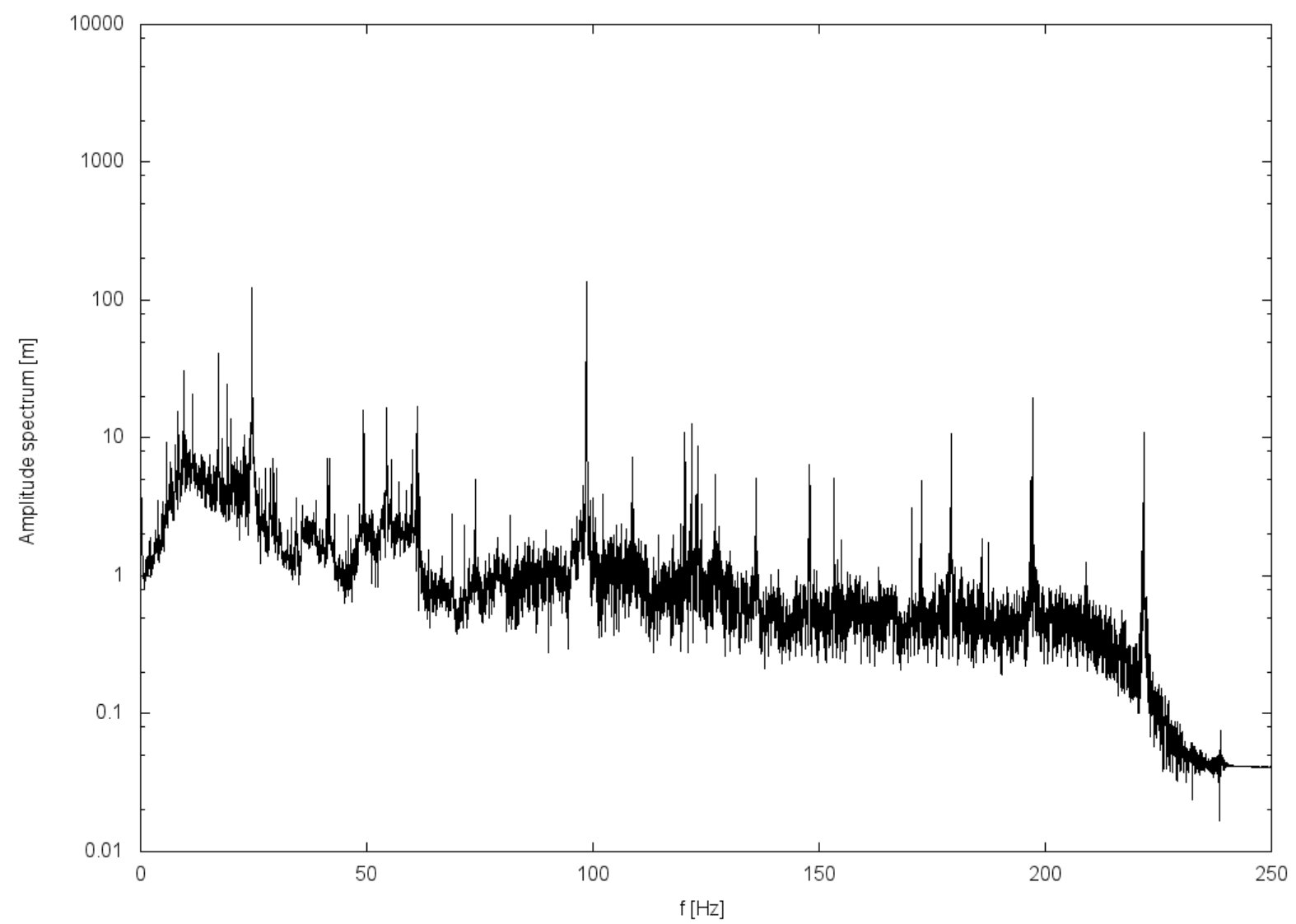

Fig. 3 Amplitude spectrum of the noise at the surface.

larger frequency (e.g. $20 \mathrm{~Hz}$ and $100 \mathrm{~Hz}$ signal) the amplitude at the receiver $x$ is attenuated to the $5^{\text {th }}$ power of the factor $e^{-\pi t^{*^{2}} f_{1}}$ at the higher frequency than at the lower frequency (which is indicated by $f_{1}$ ). For example, for a homogeneous medium with $c=$ $4000 \mathrm{~m} / \mathrm{s}, Q=100$ along trajectory of 4000 meters is $\mathrm{P}$-wave more than twelve times more attenuated at $100 \mathrm{~Hz}$ than at $20 \mathrm{~Hz}$.

Since the detectability of the events and the perforations shots depend on SNR we also investigated the spectrum of the noise at the surface measurements. A relatively increased noise level at high frequencies above $100 \mathrm{~Hz}$ at the surface geophones could possibly explain why the perforation shots were not detected by the surface monitoring. Figure 3 shows the averaged spectrum of the noise from the surface recordings. It is obvious that the noise is not stronger at frequencies above $100 \mathrm{~Hz}$ than at lower frequencies, therefore in this case the noise did not affect the detectability of the perforation shots at the surface.

Figure 4 shows another comparison of spectra of microseismic events and perforations. These spectra are observed on downhole monitoring in Marcellus shale (depths exceeding 2 kilometres). In this case, direct P-wave arrivals from perforations and microseismic events were observed on individual surface geophones, however some of the perforation shots were not visible on the surface stations. We again analyzed two microseismic events and two perforation shots. Unlike the previous case both the events and the perforations were visible on the surface geophones. Unfortunately, the surface recordings we were provided were pre-processed with an unknown filter and therefore we could not perform the spectral analysis. Hence we can show again only spectra from the borehole monitoring. The first microseismic event peaks below $100 \mathrm{~Hz}$, second one around $100 \mathrm{~Hz}$ while spectra of perforations peak between 100 and $300 \mathrm{~Hz}$ and then slowly decrease. The spectra of the microseismic events have similar patterns as in previous case, although the second event peaks at higher frequencies. The spectra of the perforation shots are very similar to the spectra in Haynesville including relatively low signal amplitudes at frequencies of $20-30 \mathrm{~Hz}$.

\section{DISCUSSION AND CONCLUSIONS}

We have shown that at least in two case studies the large microseismic events have larger amplitude at the spectral density plots than perforation or calibration shots at the frequencies below $100 \mathrm{~Hz}$. Assuming self-similarity between small and large microseismic events we can conclude that the microseismic events generally radiate energy at low frequencies. The microseismic events in the two case 


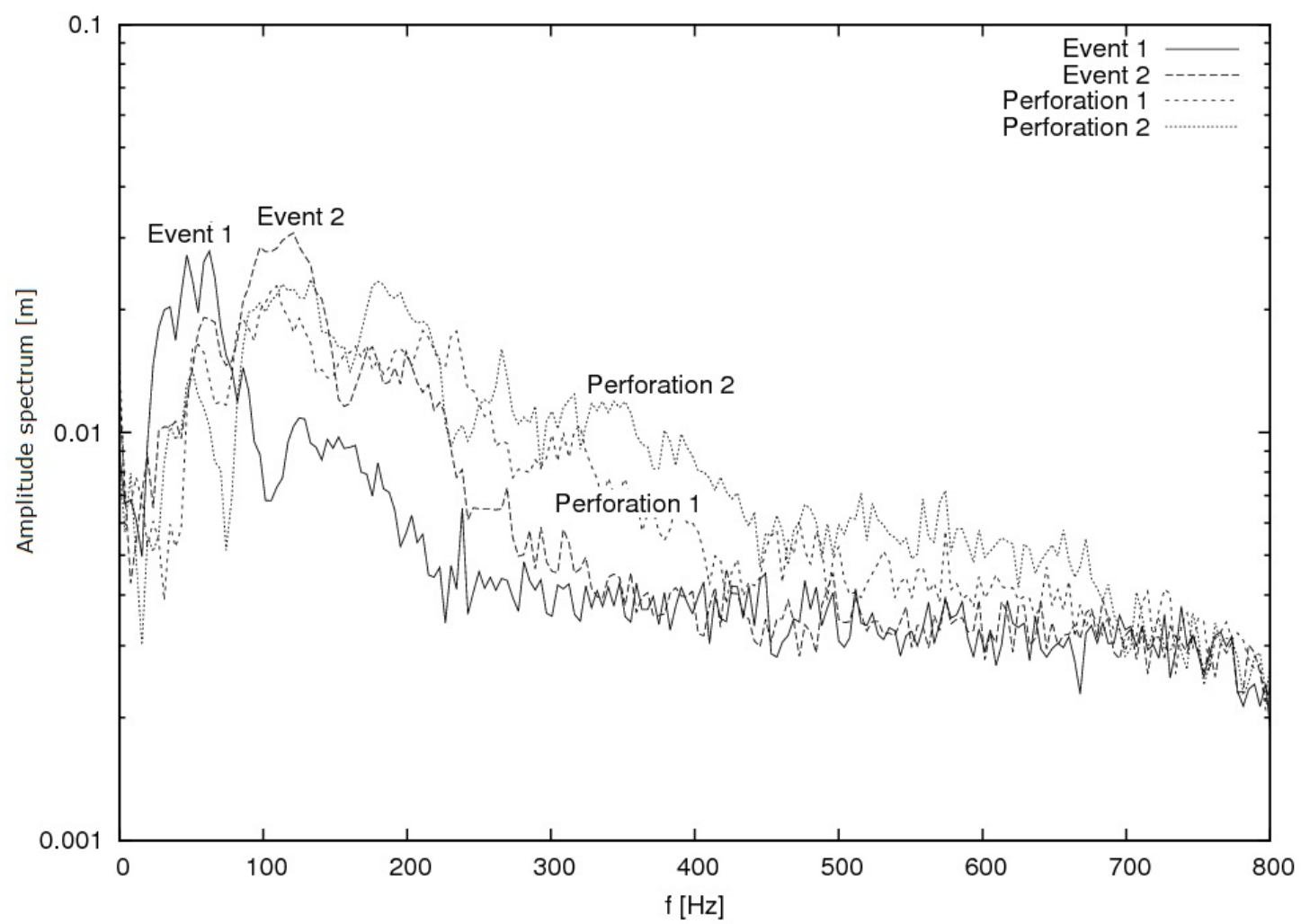

Fig. 4 Comparison of spectra of two microseismic events and two perforations from second dataset (Marcellus shale). Amplitude spectra are again averaged over all channels.

studies were selected as the largest events of the dataset to obtain optimal SNR. We have also compared the S-to-P- wave times and concluded that both microseismic events and perforation shots have approximately similar S-to-P-wave times indicating similar distance to the downhole monitoring array. Hence differences in locations relative to the monitoring array do not explain observed differences. The perforations (or string shorts) are caused by relatively short explosions and duration time is usually only several milliseconds which results in most of the energy to be radiated at frequencies above $100 \mathrm{~Hz}$. In our case studies the time delay between firing the first and the last cartridge of the gun did not exceed $0.02 \mathrm{~s}$. On the other hand, microseismic events are usually shear events resulting from permanent offset of two blocks resulting in flat amplitude spectrum of particle displacement all the way to $0 \mathrm{~Hz}$ frequency in the point source approximation (page 373-375 of Lay and Wallace, 1995). Hence for some microseismic events we can observe lower spectral amplitudes at frequencies above $100 \mathrm{~Hz}$ and higher spectral amplitudes at frequencies below $100 \mathrm{~Hz}$ relative to the perforation or string shots. As the high frequency signal is more attenuated than the low frequency signal, we may not be able to detect some of the perforation or string shots at the surface or nearsurface geophones while we are able to observe signal from microseismic events. Obviously this is not caused by coupling of the perforations to formation and, as we have shown, it cannot be explained by a increased noise level at high frequencies above 100 $\mathrm{Hz}$ at the surface geophones. Given that the frequency distribution of radiated energy is driven by the duration of the source signal we may design more detectable string shots by extending their duration, i.e. delaying individual shots.

\section{ACKNOWLEDGEMENTS}

We are grateful for permission to publish this data to Encana Oil \& Gas (USA) Inc., Shell and Microseismic Inc. This study was supported by the grant agency of the Czech Republic P210/12/2451.

\section{REFERENCES}

Aki, K. and Richards, P.G.: 2002, Quantitative seismology ( $2^{\text {nd }}$ edition), University Science Books, $700 \mathrm{pp}$.

Bardainne, T. and Gaucher, E.: 2010, Constrained tomography of realistic velocity models in microseismic monitoring using calibration shots, Geophysical Prospecting, 58, 739-753.

Bulant, P., Eisner, L., Pšenčík, I. and Calvez, J.L.: 2007, Importance of borehole deviation surveys for monitoring of hydraulic fracturing treatments, Geophysical Prospecting, 55, 891-899.

Chambers, K., Kendall, J.-M., Brandsberg-Dahl, S. and Rueda, J.: 2010, Testing the ability of surface arrays to 
monitor microseismic activity, Geophysical Prospecting, 58, 821-830.

Duncan, P. and Eisner, L.: 2010, Reservoir characterization using surface microseismic monitoring, Geophysics, 75, 75A139-75A146. DOI: 10.1190/1.3467760

Eisner, L., Gei, D., Hallo, M., Opršal, I., and Ali, M.: 2013, The peak frequency of direct waves for microseismic events. Geophysics, 78(6), A45-A49.

DOI: 10.1190/geo2013-0197.1

Lay,T. and Wallace, T.C.: 1995, Modern global seismology, Academic Press, $521 \mathrm{pp}$.

Maxwell, S., Rutledge, J., Jones, R. and Fehler, M.: 2010, Petroleum reservoir characterization using downhole microseismic monitoring, Geophysics, 75, 75A12975A137. DOI: 10.1190/?1.3477966

Rutledge, J.T., Phillips, W.S., Fairbanks, T.D. and Anderson, D.W.: 1998, Induced microearthquake patterns and oilproducing fracture systems in the austin chalk, Tectonophysics, 289, 153-169. DOI: $10.1190 / 1.1567214$

Rutledge, J.T. and Phillips, W.S.: 2003, Hydraulic stimulation of natural fractures as revealed by induced microearthquakes, Carthage Cott on Valley gas field, east Texas, Geophysics, 68, 441-452.

DOI: $10.1190 / 1.1567214$ 